\title{
Susceptibilidad erosiva asociada al proceso de reconversión agrícola productiva, cuenca semiárida de Quillota. V Región de Valparaíso, Chile
}

\author{
Erosion Susceptivility Associated with the Process of \\ Agricultural Productive Conversion in the Semiarid Catchment \\ of Quillota. V Region of Valparaiso, Chile
}

Mónica Meza Aliaga ${ }^{1}$ y Carmen Paz Castro Correa ${ }^{2}$

\section{RESUMEN}

En el marco del proceso de reconversión agrícola productiva que ha experimentado la zona norte y centro del país, se han establecido plantaciones de frutales en laderas de cerros y zonas de piedemonte; muchas veces sobrepasando los umbrales morfodinámicos aconsejables para el uso productivo. Esta práctica está otorgando a los territorios una condición de mayor susceptibilidad erosiva, influyendo también en la pérdida de calidad del suelo en los fondos de valles. Mediante el modelo de Unidades de Respuesta a la Erosión-ERU- de Märker et al. 2001, método de carácter cualitativo que arroja niveles de susceptibilidad erosiva a partir de la consideración conjunta y ponderada de las variables del sistema que influyen en la problemática, se analizó la erodabilidad en función de los patrones de ocupación de este proceso de reconversión en la cuenca semiárida de Quillota (3252'0"S $71^{\circ} 16^{\prime}{ }^{\circ}$ 'O). Los resultados arrojaron que superficies reconvertidas agrícolamente y asociadas a los sistemas de vertientes y conos aluviales obtienen una erodabilidad baja cuando poseen características de mayor densidad vegetal, menor pendiente y exposición sur, mientras que las áreas de mayor erodabilidad presentaron densidades vegetales bajas, pendientes pronunciadas y exposiciones norte. En las superficies de menor y mayor erodabilidad se hallaron prácticas de manejo como camellones a favor de la pendiente, terrazas en curvas de nivel y laderas con vegetación nativa; siendo los camellones aquellos que provocaron la mayor susceptibilidad erosiva.

Palabras clave: Unidades de Respuesta a la Erosión, prácticas de manejo, evaluación multicriterio, procesos geodinámicos.

\begin{abstract}
In the context of the agricultural productive conversion processes that have taken place in the northern and central regions of the country, fruit tree plantations have been established on hillsides and piedmont zones, often trespassing the morphodynamic thresholds advisable for productive use. This practice is granting the land a state of a greater susceptibility to erosion. By means of the model of Erosion Response Units (ERU) -Märker et al. 2001-a qualitative method that provides levels of erosion susceptibility considering the joint and analyzed variables of the system that influence this problem- the phenomenon of erodibility was examined based on the patterns of the process of conversion in the semiarid catchment of Quillota (32.52'0" $571^{\circ} 16^{\prime} 0^{\prime \prime} W$ ). The results showed that the soil -that was agriculturally converted associated to slop systems and alluvial cones-obtained low erodibility when they possess such characteristics as major vegetable density, minor gradient and south exposure; whereas the areas of major erodibility presented lower vegetable density, major gradient and north exposure. On the surfaces with minor and major erodibility handling practices like farming ridges in direction to the slope, contour terraces and hillsides with native vegetation were found; being the farming ridges those that caused the greatest erosion susceptibility.
\end{abstract}

Key words: Erosion Response Units, handling practices, multi-criterion evaluation, geodynamic processes.

\footnotetext{
1 Departamento de Ciencias Históricas y Geográficas, Universidad de Tarapacá, Av. 18 de Septiembre 2222. Arica-Chile.

2 Departamento de Geografía, Universidad de Chile, Av. Portugal 84, Santiago Centro.

* Autor por correspondencia: msmezaa@uta.cl.
}

Fecha de Recepción: 3 Mayo, 2013.

Fecha de Aceptación: 30 Agosto, 2013. 


\section{Introducción}

La degradación y erosión de suelos en varias regiones de América latina y África tiene su origen en factores sociales, económicos y culturales, que se traducen en la sobreexplotación de los recursos naturales y en la aplicación de prácticas inadecuadas de manejo de suelos y aguas (FAO, 2000).

El suelo es uno de los componentes del medio que se ve considerablemente afectado cuando no se practica un manejo adecuado. Son muchas las consecuencias de un mal manejo que repercuten directamente en el suelo; entre estas destacan la erosión, compactación, salinidad, encostramiento, disminución de fertilidad, etc., todas estas pueden ser englobadas en una sola: la degradación del suelo (Pando et al., 2003).

Mientras que muchos de estos procesos son naturales, sus impactos son agravados por sistemas inapropiados de manejo y por presiones inducidas por los seres humanos. Esto tiene como efecto la reducción del potencial productivo del suelo y de su capacidad para servir como un filtro natural o amortiguador resiliente para otros usos (Espinoza et al., 2007; Doran et al., 1994). El uso de los suelos y las prácticas de manejo marcan principalmente el grado y la dirección de los cambios en su calidad en tiempo y espacio (Quiroga y Funaro, 2004).

En el escenario del actual paradigma económico, donde las necesidades crecen cada día y aumenta la demanda por alimentos, se reproducen nuevos comportamientos relacionados al uso y manejo de los recursos, resultando paradójico el hecho de que suelos de aptitud agrícola comiencen a urbanizarse, y aquellos suelos sin dicha aptitud, como son las laderas de los cerros, se utilicen con fines agrícolas.

De acuerdo con Ruiz, 2005, en la zona central de Chile, desde la década de los ochenta se vienen realizando plantaciones de frutales y viñas en laderas de cerros y piedemontes. Esta práctica de uso del terreno en suelos de pendientes de $45^{\circ}$ o más es común en regiones con escasa pluviometría, como la III y IV Región, en donde existen considerables extensiones de plantaciones, especialmente viñas de uva pisquera y de mesa, hoy también con paltos y cítricos. En los últimos años esta superficie se ha ampliado a regiones como la III, IV, V, RM, VI y también en la $\mathrm{XV}$, cubriendo miles de hectáreas con sistemas de riego tecnificado en suelos de ladera. Considerando estas prácticas y la morfodinámica de los territorios, es interesante observar que autores como Araya-Vergara \& Börgel (1972) señalan que a partir de los $10^{\circ}$ de pendiente ya se produce erosión.

La cuenca de Quillota, localizada en la V Región de Chile (Figura 1), con características de clima semiárido templado con lluvias invernales (Errázuriz et al., 2000), ha experimentado un proceso acelerado de expansión de su frontera agrícola, escenario que se ha visto favorecido por la puesta en valor de las unidades de piedemonte en función de sus ventajas comparativas vinculadas al menor efecto de las bajas temperaturas (Gardiazabal, 2003), que permiten; gracias a técnicas de manejo como camellones y terrazas, el cultivo de frutales, particularmente paltos.

Estas nuevas formas de uso y ocupación del territorio, ligadas intrínsecamente a la apertura de las economías nacionales a los mercados externos, tiene como una de sus expresiones territoriales las fases de modernización agrícola, lo que ha generado una profunda transformación en los modos de producción y de los patrones de ocupación, donde la intervención de las cuencas ha sobrepasado los umbrales morfodinámicos normalmente aceptables respecto de los grados de pendientes, lo que puede estar influyendo negativamente en el funcionamiento del medio físico natural en general, y en la degradación morfoedafológica en particular.

Es a propósito de la masificación de estas prácticas que surge la necesidad de evaluar la vulnerabilidad erosiva que otorgan al área de estudio. Esta última, como unidad representativa del proceso de reconversión agrícola en Chile.

\section{Materiales y Métodos}

\section{Unidades de Respuesta a la Erosión}

Las Unidades de Respuesta a la Erosión (ERU) corresponden a un modelo cualitativo que define unidades homogéneas de erosión y su vulnerabilidad natural frente a procesos erosivos, lo que se representa en un mapa de susceptibilidad erosiva para la cuenca de Quillota que posee una superficie de $432,2 \mathrm{~km}^{2}$ y se ubica a la altura de la comuna de La Calera y en toda el área de influencia que ejerce en este sector el río Aconcagua, que se constituye en un amplio y fértil valle hasta el cordón del cerro San Pedro por el sur (Figura 1).

Märker et al. (2001) define las ERU como "la distribución de las unidades tridimensionales del terreno, que están heterogéneamente estructuradas; 


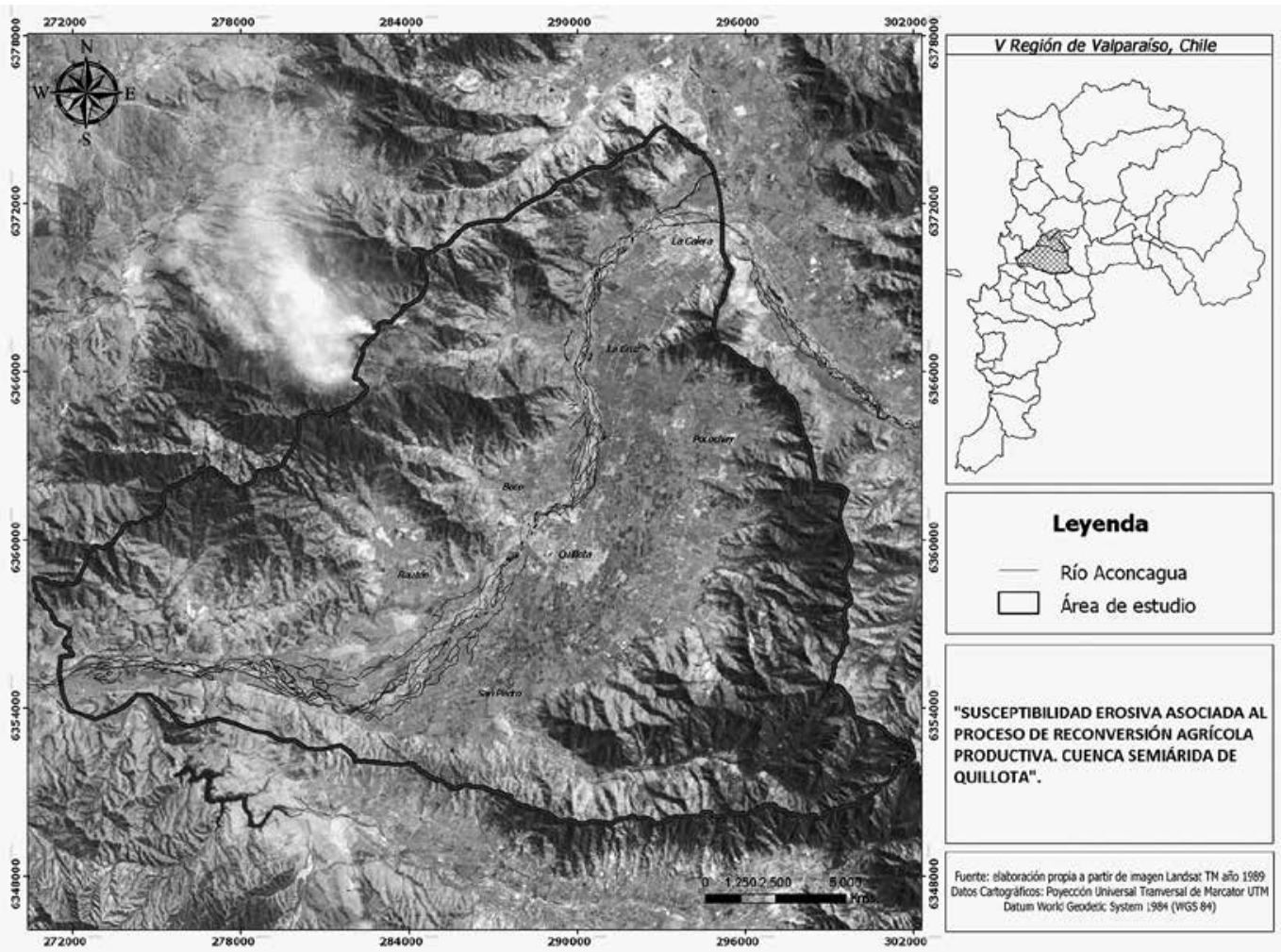

Figura 1. Área de estudio, cuenca de Quillota, sección media-baja de la cuenca del Aconcagua, V Región, Chile.

cada una de ellas posee características homogéneas en cuanto a los procesos dinámicos de erosión, pero con una variación ligera dentro de la unidad al compararlo con sus vecinos. Ellas están controladas por sus propiedades fisiográficas y el manejo de su ambiente natural y humano".

Las unidades homogéneas de erosión (ERU) resultan de la superposición entre las diferentes capas de información físico-natural; distinguiendo la erosividad del sistema que depende de la precipitación y por otro la erodabilidad ligada a las características del terreno, como el uso del suelo, la geología y la geomorfología.

Al reclasificar y recubrir las capas de información pertinentes, utilizando tecnología SIG, se obtienen Unidades de Respuesta que consideran la heterogeneidad fisiográfica y antropogénica de la cuenca del río (Märker et al., 2001) (Figura 2).

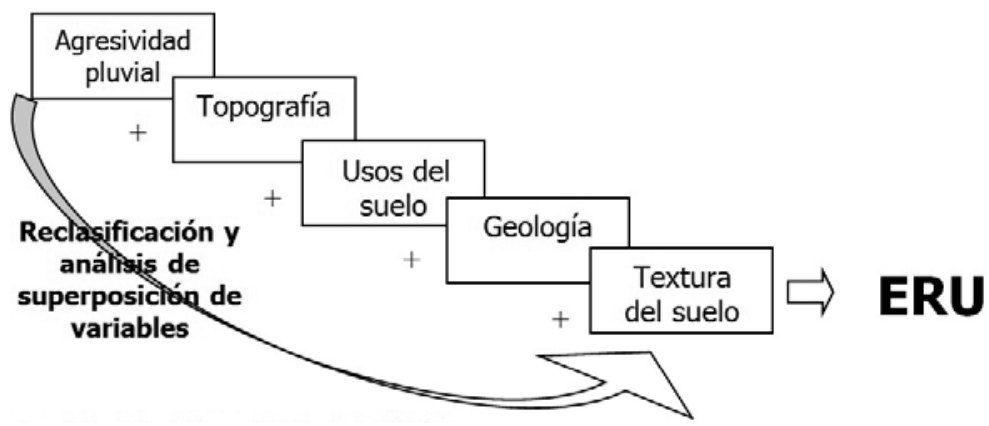

Fuente: Adaptado de Marker et al. (2001).

Figura 2. Procedimiento de superposición de las variables utilizadas para la determinación de las ERU. 
Las variables seleccionadas para describir los atributos fisiográficos que influyen en los procesos de erosión fueron seleccionadas de acuerdo con su condición de factor interno y externo de la erosión, de modo que dentro de las primeras se encuentran la topografía (pendiente y exposición) determinadas por medio de un Modelo de Elevación Digital (DEM) con curvas cada $25 \mathrm{~m}$, usos de suelo obtenidos y actualizados del Plan Intercomunal de Valparaíso Satélite-Quillota año 2004, la geología del área de estudio extraída de la carta geológica del Sernageomin de 1997 y la textura del suelo del Estudio Agrológico de la V Región de CIREN año1997. Por su parte, el factor externo vinculado a la agresividad pluvial del área de estudio fue obtenido gracias al cálculo del Índice Modificado de Fournier (IMF), con datos de las estaciones pluviométricas Lo Rojas, Quillota, Los Aromos, quebrada Alvarado y estero Rabuco de la DGA. Según los resultados de exposición a la precipitación y pendientes, la variable agresividad pluvial fue adaptada a la topografía del área de estudio.

Debido a la naturaleza de cada variable se sistematizaron y reclasificaron en función de los niveles de susceptibilidad erosiva intrínsecos de cada una de ellas y utilizando los estudios de Jordan (2000) para la variable agresividad pluvial, patrones de umbrales geomorfológicos de Araya-Vergara \& Börgel (1972) para pendientes, Lagos (2005) para la exposición a la precipitación, Kunhi y Pfiffner (2001) para la geología, Henríquez et al. (2006) en usos de suelo y Morgan (1997) para la textura del suelo.

Cada variable trabajada en formato ráster se utilizó en el proceso de superposición final.

\section{Método de Evaluación Multicriterio (EMC)}

Previo al procedimiento de superposición de variables, fue fundamental representar jerárquicamente el problema. La técnica utilizada fue el análisis multicriterio que permite asignar pesos relativos a las variables que participan del modelo. La metodología utilizada se denomina Proceso Analítico Jerárquico (AHP) desarrollada por Saaty (1997), con ella es posible modelar variables tangibles e intangibles, cualitativas y cuantitativas, de modo de organizar la información y conocimiento en una estructura analítica jerárquica.

Estructurada la jerarquía, se efectuó un proceso de priorización; la priorización involucró la obtención de opiniones en respuesta a preguntas sobre el predominio de un elemento sobre otro cuando se compara respecto de una propiedad, en este caso, la propiedad dice relación con cuánto más influye en la susceptibilidad erosiva una variable respecto de otra, asignando un valor de acuerdo con la escala fundamental para comparaciones por pares de Saaty (1997).

Como resultado se obtiene un peso para cada variable y subvariable siendo coherente cuando la inconsistencia arrojada por el modelo es menor a 0,1 . La información fue procesada en el software Expert Choice 11.

Para la obtención de las ERU se superpusieron y adicionaron las variables cuyo valor de píxel corresponde a los pesos obtenidos con la EMC. Se verificó que el rango de valores tuviera una distribución normal y se reclasificó con la medida estadística desviación estándar, de modo que a partir de la media se obtuvieron los menores y mayores valores de susceptibilidad erosiva.

\section{Validación del modelo y Análisis de correlación}

Se determinó la intensidad de relación existente entre las ERU y cada una de las variables escogidas para explicar la susceptibilidad erosiva mediante un análisis de correlación lineal simple calculando el coeficiente de correlación.

La variable dependiente es la variable que se predice o calcula, para este estudio las variables dependientes corresponden a la agresividad pluvial, pendiente, exposición, usos del suelo, geología y textura. La variable independiente es la variable que se desea explicar y proporciona las bases para el cálculo; quedando representada por las ERU.

El coeficiente de correlación (r) es un número real comprendido entre -1 y 1 . Si el coeficiente de correlación lineal toma valores cercanos a 1 la correlación es firme y directa y será tanto más sólida cuanto más se aproxime r a 1. La operación se realizó en el programa Idrisis Andes mediante su módulo GIS Analysis.

Con el propósito de validar el modelo, se contrastaron los resultados obtenidos con la situación actual del área de estudio. Para ello, la aproximación se realizó comparando las unidades territoriales que arrojaron Altas y Bajas susceptibilidades erosivas, con imágenes Google Earth año 2012 y visitas a terreno. 


\section{Resultados y Discusión}

La agresividad pluvial obtenida mediante el IMF exhibe rangos que van desde 139,6 a 196,4 mm, los que de acuerdo con la clasificación CEC, 1992, presentada por Jordan, 2000, describen áreas de Alta y Muy alta erosividad que aumentan su valor de precipitación en la medida que el terreno se hace más escarpado en la cuenca.

Las pendientes obtenidas por medio del DEM y su posterior clasificación revelan que el área de estudio posee una fisiografía bastante diversa, con rangos de pendientes que fluctúan entre $0^{\circ}$ en el fondo de valle y $87^{\circ}$ en los sectores de mayor altitud en la divisoria de aguas que limita la cuenca. Respecto de la variable exposición, la mayor parte del área de estudio exhibe una susceptibilidad erosiva muy alta asociada al norte plano o cenital que es impactado verticalmente por las gotas de lluvia. Aquellas superficies de susceptibilidad erosiva baja se localizan en las zonas de sombra pluviométrica asociadas a las laderas de exposición sur, sureste y suroeste. Desde el punto de vista erosivo, los usos de suelo generan determinado grado de protección al suelo (Morgan, 1997) u originan transformaciones vinculadas principalmente al aumento de su impermeabilización (Henríquez et al. 2006). Como la superficie en la que se emplaza el río Aconcagua y el área urbanizada de la ciudad de Quillota presentan una dinámica distinta; asociadas respectivamente a los procesos fluviales de excavación y deposición, y a procesos degradativos no erosivos (Casanova et al., 2006) vinculados a la impermeabilización del recurso suelo, fueron excluidas en la determinación de la variable usos del suelo. El área de estudio históricamente ha presentado condiciones favorables para la agricultura, tanto desde el punto de vista de su posición morfoclimática como de la característica de sus suelos, por lo que la variable usos del suelo indica que la categoría con mayor representatividad espacial corresponde a los terrenos agrícolas que cubren el $72,2 \%$ del área de estudio; siendo los camellones a favor y en contra de la pendiente y las terrazas las prácticas de manejo agrícola más utilizadas. Cabe señalar en cuanto a las prácticas de manejo que el palto (Persea americana Mill), principal variedad que ha poblado las laderas de Quillota, no necesita un suelo demasiado profundo para su enraizamiento, pues al tener raíces superficiales produce abundantes cosechas en suelos de 30 a $40 \mathrm{~cm}$ de profundidad, siempre y cuando tenga un subsuelo de excelente drenaje (Gardiazabal, 2004). Su cultivo en el área de estudio ha sido posible gracias a las mencionadas prácticas de manejo con que se interviene el terreno $\mathrm{y}$, en este sentido, resulta interesante mencionar el trabajo de Youlton (2005), que cuantificó la erosión en camellones construidos en laderas del valle de Quillota, y reconoció, mediante mediciones experimentales in situ, que los camellones recién construidos y sin cobertura vegetal generan una pérdida de suelo 650 veces mayor respecto de un área inalterada y cubierta por vegetación natural, existiendo correlación entre estos resultados y los niveles de susceptibilidad erosiva arrojados por el modelos de las ERU en las unidades de significativa intervención.

Desde el punto de vista geológico, el área de estudio está compuesta por secuencias sedimentarias, secuencias volcánicas y rocas intrusivas, que de acuerdo con Kunhi y Pfiffner (2001) es posible clasificar en vulnerabilidades erosivas alta, media y baja, respectivamente.

La textura del suelo en el área de estudio está dominada por la influencia de suelos francos, los que en términos generales son los más adecuados para la práctica de la agricultura y fluctúan entre una baja y alta susceptibilidad erosiva, representando el $88,92 \%$ del área de estudio.

\section{Análisis jerárquico y EMC}

La organización de cada una de las variables con su respectiva categoría de susceptibilidad erosiva se realizó mediante la construcción de una estructura jerárquica gracias al proceso de priorización que involucró la consulta a expertos. Con una inconsistencia aceptable de 0,05, la EMC permitió conocer el grado de participación de cada una de las variables seleccionadas. En la Tabla 1 se presenta la estructura jerárquica y los pesos o ponderaciones obtenidas y asignadas a las variables con que se modelaron las clases de erodabilidad.

\section{Análisis por clase de ERU}

Las clases de susceptibilidad erosiva obtenidas para la cuenca de Quillota se presentan en la Tabla 2, que muestra las superficies del área de estudio por cada clase de erodabilidad de acuerdo a su rango de clasificación. Para el análisis y elaboración del mapa final (Figura 3) se excluyó el área urbanizada y la caja del río por responder a dinámicas físiconaturales particulares. 
Tabla 1. Estructura jerárquica y categorías según porcentaje de participación y peso de cada variable constituyente del modelo ERU

\begin{tabular}{|c|c|c|c|}
\hline Variable/Subvariable & Categoría & Porcentaje & Peso \\
\hline \multirow[t]{3}{*}{ Agresividad pluvial } & & 50,6 & \\
\hline & Alta & 33,3 & 0,16850 \\
\hline & Muy alta & 66,7 & 0,33750 \\
\hline Topografía & & 24,3 & \\
\hline \multirow[t]{6}{*}{ Pendiente } & & 66,7 & \\
\hline & Muy baja & 6,1 & 0,01482 \\
\hline & Baja & 9,7 & 0,02357 \\
\hline & Moderada & 16 & 0,03888 \\
\hline & Alta & 26,3 & 0,06391 \\
\hline & Muy alta & 41,9 & 0,10182 \\
\hline \multirow[t]{5}{*}{ Exposición } & & 33,3 & \\
\hline & Baja & 9,6 & 0,02333 \\
\hline & Moderada & 16 & 0,03888 \\
\hline & Alta & 27,7 & 0,06731 \\
\hline & Muy alta & 46,7 & 0,11348 \\
\hline \multirow[t]{6}{*}{ Usos de suelo } & & 10,7 & \\
\hline & Baja & 6,1 & 0,00653 \\
\hline & Baja - media & 9,7 & 0,01038 \\
\hline & Media & 16 & 0,01712 \\
\hline & Media - alta & 26,3 & 0,02814 \\
\hline & Alta & 41,9 & 0,04483 \\
\hline \multirow[t]{4}{*}{ Geología } & & 7,9 & \\
\hline & Baja & 16,3 & 0,01288 \\
\hline & Media & 29,7 & 0,02346 \\
\hline & Alta & 54 & 0,04266 \\
\hline \multirow[t]{5}{*}{ Textura } & & 6,5 & \\
\hline & Muy baja & 9,5 & 0,00618 \\
\hline & Baja & 16 & 0,01040 \\
\hline & Moderada & 27,7 & 0,01801 \\
\hline & Alta & 46,7 & 0,03036 \\
\hline Índice de inconsistencia & & & \\
\hline
\end{tabular}

Fuente: Elaboración propia.

Clase ERU 1: Se encuentra distribuida principalmente en los conos aluviales y en el sistema de vertientes volcánicas, representando solo el 2,2\% de la superficie. Se caracteriza por la combinación de condiciones de susceptibilidad erosiva baja y media en cada una de las variables individuales, excepto en la agresividad pluvial que es alta en todas las formas de acumulación del área de estudio. Se explica además por qué las vertientes volcánicas asocian vegetación nativa dispersa y plantaciones forestales; mientras que los conos aluviales, vegetación nativa dispersa y plantaciones de árboles frutales desarrollados.
Clase ERU 2: En los conos aluviales esta clase es atribuible a su baja vulnerabilidad erosiva por concepto de pendiente y texturas francas, principalmente. Mientras en el fondo de valle destaca su pendiente horizontal y esencialmente texturas francas a franco-arcillosas, las que varían entre baja y moderada susceptibilidad erosiva. Se les atribuye una media vulnerabilidad a la erosión en relación con el uso del suelo, que en su mayoría corresponde a terrenos agrícolas con cultivos anuales y es la clase de susceptibilidad con mayor representatividad espacial del área de estudio $\left(159,4 \mathrm{~km}^{2}\right)$. 
Tabla 2. Superficie del área de estudio por clase de Unidades de Respuesta a la Erosión (ERU)

\begin{tabular}{ccccrr}
\hline Clase ERU & Susceptibilidad erosiva & Rangos de clasificación & Desviación estándar & $\mathrm{km}^{2}$ & $\%$ \\
\hline 1 & Muy baja & $0,28118-0,42783$ & $<-1,5--0,5$ & 9,7 & 2,2 \\
2 & Baja & $0,42784-0,55622$ & $-0,50-0,50$ & 159,4 & 36,9 \\
3 & Media & $0,55623-0,68461$ & $0,50-1,50$ & 123,8 & 28,7 \\
4 & Alta & $0,68462-0,81299$ & $1,5-2,5$ & 48,7 & 11,3 \\
5 & Muy alta & $0,81300-0,89033$ & $>2,5$ & 49,9 & 11,5 \\
No aplica (caja de río, área urbana) & & & 40,7 & 9,4 \\
\hline
\end{tabular}

Fuente: Elaboración propia.

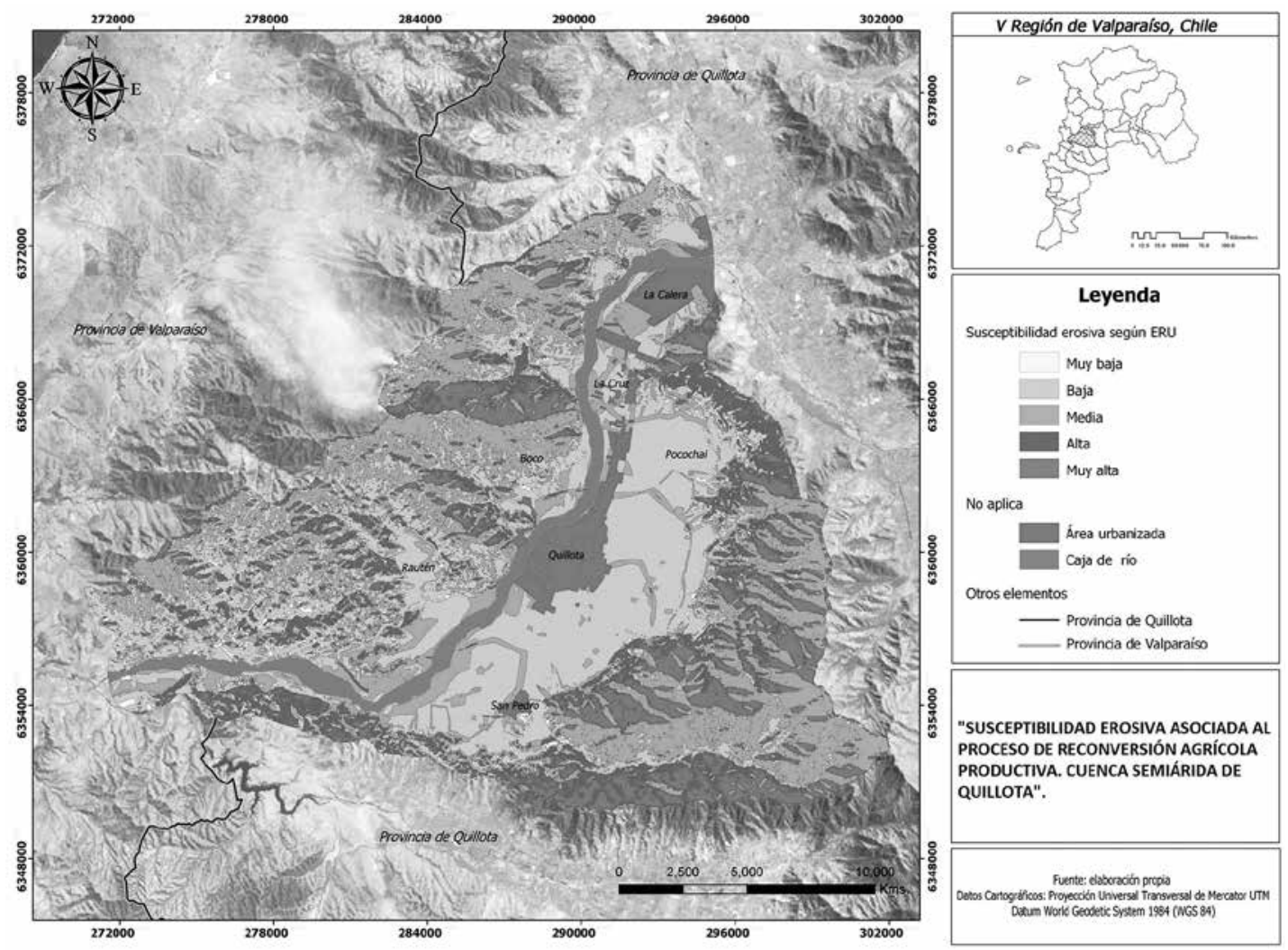

Figura 3. Unidades de Respuesta a la Erosión, cuenca de Quillota, V Región, Chile.

Clase ERU 3: Se encuentra asociada al sistema de vertientes, fundamentalmente a las laderas de exposición suroeste, sur y sureste. Posee una amplia representatividad espacial, con 123,8 km 2 equivalentes al 28,7\% del área de estudio, y se caracteriza por pendientes pronunciadas, muy alta agresividad pluvial y baja vulnerabilidad por exposición; esta última variable sirve de regulador de la vulnerabilidad erosiva en esta clase, porque permite el desarrollo de vegetación nativa, principalmente en los talwegs torrenciales, lo que contribuye a estabilizar las vertientes y controlar la erodabilidad, ya que a la vez la susceptibilidad erosiva vinculada a la geología también es alta al estar asociada a vertientes monoclinales en secuencias sedimentarias. En el fondo de valle, adyacente a los sectores urbanos, esta clase aparece relacionada a texturas franco-arenosas finas. 
Clase ERU 4: Esta clase se encuentra sujeta principalmente al sistema de vertientes monoclinales antropizadas, tanto marinas como continentales, coincidiendo con aquellos sectores mayormente intervenidos con frutales en laderas de cerro, tanto con camellones como con terrazas. Se caracterizan por la combinación de susceptibilidades erosivas altas a muy altas en relación con las variables pendiente, exposición y geología. Posee en toda su distribución muy alta agresividad pluvial. Los usos de suelo corresponden, por un lado, a aquellos de medianas susceptibilidades erosivas relacionadas con la categoría de terrenos agrícola y plantaciones frutales en ladera de cerro, y por otro, a las áreas de escasa vegetación o de coberturas menores al $25 \%$. Esta clase posee una superficie de $48,7 \mathrm{~km}^{2}$, representando $11,3 \%$ del área evaluada.

Clase ERU 5: Presenta una superficie de $49,9 \mathrm{~km}^{2}$, con vulnerabilidades baja y media en las variables uso del suelo y textura, pues la agresividad pluvial, pendiente, exposición y geología al ser altas y muy altas, le otorgan a estas unidades la característica de mayor susceptibilidad erosiva del área de estudio. Se distribuye principalmente en el sistema de vertientes monoclinales marinas y continentales de exposición norte. Respecto de los usos de suelo, esta clase incorpora las áreas de escasa vegetación y las que poseen algún grado de degradación, además de algunos sectores de vertientes intervenidas con camellones a favor de la pendiente y plantaciones recientes.

\section{Validación del Modelo y Análisis de correlación}

Se escogieron sectores con resultados de susceptibilidades erosivas altas y bajas. El primero coincide con unidades que constituyen evidencia de la expansión de la frontera agrícola en el escenario de reconversión productiva que ha experimentado el área de estudio, es decir, sectores de ladera de cerro con alta intervención antrópica cultivados con frutales en pendientes por sobre $\operatorname{los} 10^{\circ}$. El segundo sector, igual que el anterior, es representativo de sectores con intervención antrópica, pero debido principalmente a su baja pendiente y alta cobertura vegetal arrojaron susceptibilidades erosivas bajas. Los resultados fueron además corroborados en campañas de terreno.

Susceptibilidades erosivas altas como las obtenidas para el sector del cordón de San Pedro a la altura de la zona urbana de la comuna de La Cruz coinciden con superficies en laderas de cerro intervenidas con fines agrícolas. De lo anterior es posible señalar que unidades territoriales que presentan intervenciones en camellones y terrazas con árboles frutales, a pesar de estar cubiertas con vegetación, si esta última corresponde a árboles pequeños de escaso desarrollo y follaje, tenderán a comportarse bajo condiciones de alta susceptibilidad erosiva.

Dichas unidades territoriales con pendientes sobre los $10^{\circ}$ podrían asociarse, en general, a modelos explicativos que responden a procesos dinámicos de erodabilidad con coberturas vegetacionales baja y media, alta agresividad pluvial, pendiente de $10,1^{\circ}$ a mayores de $20^{\circ}$, exposición NO, NE y norte plano, uso de suelo con plantación frutal, rocas sedimentarias marinas litorales y continentales, texturas francas, franco-arcillo-limosa y franco-arcillo-arenosa.

Por su parte, las susceptibilidades erosivas bajas responden fundamentalmente a la densidad vegetacional que albergan estas unidades, pues en la subcuenca de Rautén fue posible constatar dicha situación. Los resultados de las ERU en esta unidad dan cuenta de procesos dinámicos con coberturas vegetacionales alta y muy alta, alta agresividad pluvial, pendientes de $0^{\circ}$ a $5^{\circ}$ y en la cabecera de la subcuenca entre $10,1^{\circ}$ y $20^{\circ}$, exposición SO, S, $\mathrm{SE}$ y en ciertos ángulos $\mathrm{NO}$ y NE, uso de suelo con cultivos anuales y plantación frutal, rocas sedimentarias continentales y depósitos de origen antrópico, texturas francas, franco-arenosa muy fina, franco-arcillo-arenosa y arcillo-limosa.

Las ERU son explicadas según el grado de correlación existente entre la susceptibilidad erosiva y cada una de las variables consideradas. En orden de mayor a menor valor de correlación el resultado por variable se presenta en la Tabla 3.

Tabla 3. Coeficiente de correlación por variable

\begin{tabular}{lc}
\hline Variable independiente & Coeficiente \\
\hline Agresividad pluvial & 0,42 \\
Pendiente & 0,36 \\
Exposición & 0,21 \\
Geología & 0,07 \\
Uso del suelo & 0,04 \\
Textura del suelo & 0,03 \\
\hline
\end{tabular}

Fuente: Elaboración propia. 
La relación de dependencia entre la susceptibilidad erosiva del área de estudio y las variables consideradas en la obtención de las ERU es más marcada para las variables agresividad pluvial, pendiente y exposición; pues la agresividad pluvial es significativa, ya que la erosividad, que representa uno de los factores externos más importantes de la erosión, desencadena la pérdida del suelo en la medida que la precipitación golpea la superficie del suelo y disgrega sus partículas, quedando estas últimas susceptibles de ser arrastradas.

Los valores obtenidos son de carácter explicativo, no predictivos, pues el coeficiente de correlación no es la medida más adecuada para evaluar la predicción de un modelo, de modo que los valores obtenidos muestran que las tres variables que alcanzaron los mayores coeficientes de correlación son las más significativas dentro del modelo, y que la geología y textura, a pesar de su importancia como atributos intrínsecos del suelo que influyen en su erodabilidad, probablemente merecen un tratamiento a escala local que por el alcance de este estudio no fueron incorporadas, obteniendo un menor grado de significación al igual que los usos del suelo.

En relación con la aplicación del modelo ERU y su contraste con la dinámica actual de erosión, surge la hipótesis de que gran parte de las unidades de erosión corresponden a formas relictas de épocas climáticas semiáridas y áridas anteriores, donde hubo precipitaciones significativas de carácter torrencial que provocaron la erosión lineal presente. Bajo el clima actual, la dinámica morfológica de estas formas se estabilizó y posee una importante cobertura de vegetación nativa, aunque producto del uso agrícola intensivo en las últimas tres décadas y de la variabilidad climática existente, es probable esperar una nueva dinamización de dichas formas erosivas.

Según Morgan (1997), una zona de alto riesgo de erosión la constituyen justamente las áreas donde el relieve y los suelos asociados son consecuencia de un clima anterior. Considerando este escenario, la respuesta del suelo a los cambios de la cobertura vegetal ocasionados por la acción humana, como los ocurridos en el área de estudio, pueden depender notablemente de la herencia geomorfológica derivada de condiciones climáticas previas.

\section{Conclusión}

La evaluación cualitativa de la susceptibilidad erosiva de los suelos del área de estudio representan las condiciones actuales debidas al accionar conjunto de los procesos naturales y aquellos inducidos por el uso y manejo agrícola. El intento por modelar las áreas más vulnerables a la erosión arroja resultados de susceptibilidad erosiva, donde la tendencia es que los sectores de alta erodabilidad se encuentran asociados a los sistemas de vertientes y conos aluviales, con pendientes pronunciadas y exposiciones norte, coincidiendo con aquellas zonas de alta intervención agraria como el cordón de cerros San Pedro. Los sectores de menor susceptibilidad erosiva, como es el caso de la subcuenca de Rautén en la comuna de Quillota, a pesar de mostrar un alto grado de intervención, posee características vinculadas a su menor pendiente, exposición sur y mayor densidad de cobertura vegetacional, que se traducen en una menor erodabilidad actual.

Las variables utilizadas para la determinación de las ERU, que influyen en la erodabilidad, son de carácter universal, no obstante, en un país con las características geomorfológicas de Chile y con una variabilidad topográfica extremadamente alta, surge la necesidad de realizar análisis locales que implican adaptar y complejizar el análisis, considerando otros factores como son el tipo de suelo, la consolidación de los materiales superficiales y, en este caso también, el tipo de manejo del suelo.

Por su parte, los grados de erodabilidad obtenidos también dan cuenta del efecto que ha tenido la ampliación de la frontera agrícola y podría explicarse por lo agresivo de las técnicas de manejo, que no consideran la protección actual del recurso suelo en función de sus características naturales y procesos dinámicos.

\section{Agradecimientos}

Se extienden los agradecimientos al proyecto FONDECYT No 1071098 "Vulnerabilidad y Niveles de Degradación del Sistema Morfoedafológico a las Transformaciones Productivas y Expansión Urbana. Análisis integrado y comparativo de cuencas", por su aporte con información base y al Convenio de Desempeño Universidad de Tarapacá-Ministerio de Educación. 


\section{Literatura Citada}

Araya-Vergara, J.f. \& Borgel.

1972. Definición de Parámetros para Establecer un Banco Nacional de Riesgos y Amenazas Naturales. Criterios para su Diseño. ONEMI/PNUD Uchile/92/009/A. Chile, pp. 219.

Casanova, M., Leiva, C., Seguel, O., Salazar, S. y Luzio W. 2006. Degradación de suelos y desertificación, pp. 353-383. En: Avances en el conocimiento de los suelos de Chile. Luzio W. y Casanova, M. (Eds.). Santiago, Chile, pp. 393. Centro de Información de Recursos Naturales (CIREN). 1997. Estudio Agrológico V Región, Santiago, Chile, pp. 366 Doran, J.W. y Parkin, B.T.

1994. Defining Soil Quality for a Sustainable Environment. Soil Science Society of America, Inc. Special Publication. $N^{\circ}$ 35. Madison, Wisconsin, USA.

Errázuriz, A. M., Cereceda, P., González, J.I., González, M., Henríquez, M., Rioseco, R.

2000. Manual de Geografía de Chile. Ed. Andrés Bello, pp. 443.

Espinoza, Y. y Malpica, L.

2007. Mediciones simples para evaluar el estado de la calidad y salud del suelo bajo pasturas. INIA-CENIAP. Maracay, Venezuela.

Organizacion de las Naciones Unidas para la Alimentación y

la Agricultura (FAO).

2000. Manual de prácticas integradas de manejo y conservación de suelos. Boletín $\mathrm{N}^{\circ} 8$ de tierras y aguas, pp. 201.

Gardiazabal, F.

2003. $45^{\circ}$ de inclinación, y no hay problema. Revista avance agrícola. 13 (118) 10-11. Quillota, Chile.

Gardiazabal, F.

2004. Factores agronómicos a considerar en la implantación de un huerto de paltos, pp. 1-17. En: Sociedad Gardiazabal y Magdahl. $2^{\circ}$ Seminario Internacional de Palto. Quillota, Chile.

Henríquez, C., Azócar, G. y Aguayo, M.

2006. Cambio de uso del suelo y escorrentía superficial: aplicación de un modelo de simulación espacial en Los Ángeles, VIII Región del Biobío, Chile. Revista de Geografía Norte Grande. 36: 61-74. Santiago, Chile.

Jordán, A. y Bellinfante, N.

2000. Cartografía de la erosividad de la lluvia estimada a partir de datos pluviométricos mensuales en el campo de Gibraltar (Cádiz). Rev. Edafología. Vol. 7-3, pp. 83-92. Sevilla, España.
Lagos, M.

2005. Protocolo para la selección de alternativas para la conservación de suelos en laderas. Serie técnica No 18 . Servicio Agrícola y Ganadero. Santiago. Chile, pp. 78.

Märker M., Moretti S. \& Rodolfi G.

2001. "Integrated Water Resources Management System", Capítulo "Assessment of Water Erosion Processes and Dynamics in Semi-arid Regions of Southern Africa (kwazulu / Natal, RSA, and Swaziland) Using the Erosion Response Units Concept (ERU's)". Revista Geogr. Fis. Dinam. Quat. Vol. 24. Issue 1. Florencia, Italia, pp. 71-84.

Morgan, R.P.C.

1997. Erosión y Conservación del Suelo. Ediciones MundiPrensa. Madrid, España, pp. 343.

Pando, M., Gutiérrez, M., Maldonado, A., Palacio, J.L. y

Estrada, E.

2003. Comparación de Métodos en la estimación de erosión hídrica. Investigaciones Geográficas Universidad Nacional Autónoma de México. № 051. México, pp. 23-36.

Quiroga, A. y Funaro, D.

2004. Materia Orgánica. Factores que condicionan su utilización como indicador de calidad de mollisoles, de las regiones semiárida y subhúmeda pampeana. XIX congreso argentino de la ciencia del suelo. Actas, pp. 476.

Ruiz, C. (ED.).

2005. Manual de especificaciones técnicas de buenas prácticas de manejo de suelos en laderas. Serie técnica $N^{\circ} 20$. Servicio Agrícola y Ganadero, SAG. Santiago, Chile, pp. 56.

Saaty, T.

1997. Toma de decisiones para líderes: El proceso analítico jerárquico en la toma de decisiones en un mundo complejo. Traducción de Escudey M., Martínez E., Vargas L. RWS Publications, pp. 429.

Servicio Nacional de Geología y Minería.

2002. Mapa Geológico de Chile. Serie Geológica Básica. $\mathrm{N}^{\mathrm{o}} 75$. Hoja 2.

Youlton, C.

2005. Cuantificación de la erosión en camellones a favor de la pendiente para el cultivo frutal de laderas en el valle de Quillota, V Región, Chile. Taller de Licenciatura Ing. Agr. Quillota. Universidad Católica de Valparaíso, Facultad de Agronomía. Chile, pp. 66. 